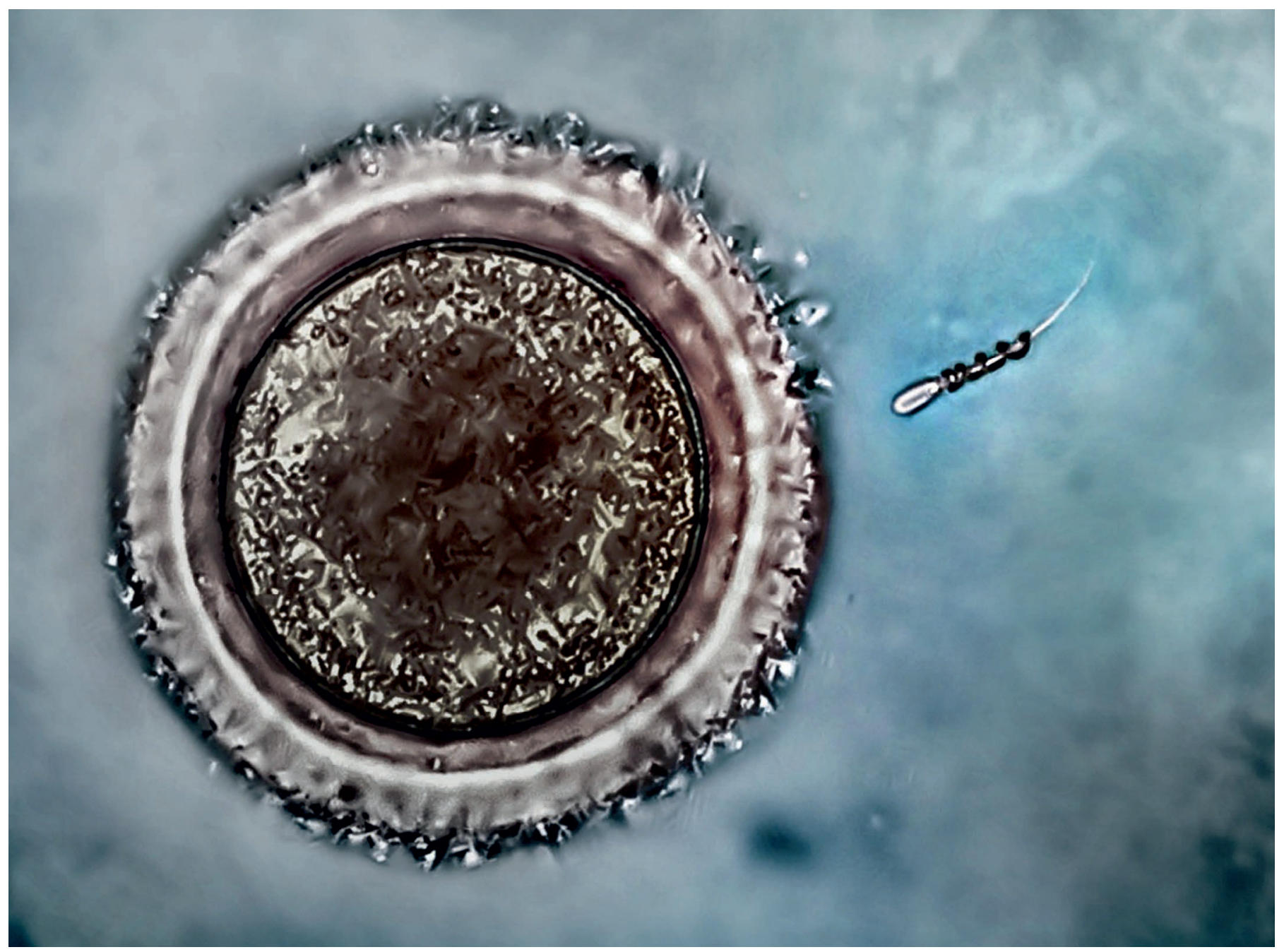

A helical micromotor helps an immotile but healthy bovine sperm cell get to an egg in culture.

\title{
Medical microbots need better imaging and control
}

Mariana Medina-Sánchez and Oliver G. Schmidt set priorities for more realistic tests of tiny machines that could be used to diagnose and treat conditions.

$\mathrm{M}$ ore than 50 years ago, physicist Richard Feynman spoke of "swallowing the surgeon" in his classic lecture, 'There's plenty of room at the bottom. Today, scientists are designing microscopic devices - microbots and micromotors - to eventually move through the body to perform medical tasks. Synthetic rods, tubes, helices, spheres or cages as small as a cell could be sent into the blood, liver, stomach or reproductive tract to diagnose conditions, carry drugs or perform surgery.
So far, most microbot experiments have been done in vitro under conditions very different from those in the human body. Many devices rely on toxic fuels, such as hydrogen peroxide. They are simple to steer in a Petri dish, but harder to control in biological fluids full of proteins and cells, and through the body's complex channels and cavities.

To enter clinical trials, microbots must clear two major hurdles. First, researchers need to be able to see and control them operating inside the body - current imaging techniques have insufficient resolution and sensitivity. Second, the vehicles need to be biocompatible and be removed or stabilized after use. Achieving both aims would set the stage for further improvements - in steering and mobility, materials and capabilities.

We call on microrobotics researchers, materials scientists and bioimaging and medical specialists to work together to solve these problems. And regulatory agencies need to put in place directives for testing therapeutics that are based on microbots. 
There are three types of micromotors. They can be categorized according to their main propulsion mode: chemical, physical or biological (see 'Three micromotor prototypes'). Each has pros and cons.

Chemical micromotors transform fuel energy into motion ${ }^{1}$. Often, a catalyst (such as platinum, silver or palladium) within the micromotor reacts with liquid surrounding it (usually hydrogen peroxide or organic compounds). These motors are hard to control. Some move by expelling gas bubbles from one end of an asymmetrical tube. Others are made of two metals (usually gold and platinum) and propelled by differences in, for instance, tension, fuel consumption or light absorption rates between their faces. They may be guided by chemical or thermal gradients in their surroundings, or by applying magnetic fields, light or ultrasound.

Outside the body, micromotors can be based on poisonous fuels. For example, they could burn a pollutant in water as fuel, or be used for on-chip chemical and biological sensing. For in vivo uses, they need to co-opt fuels that are present in the body, such as glucose, urea or other physiological fluids ${ }^{2}$. For example, tubular micromotors have been propelled by dissolving zinc in acid in a mouse's stomach ${ }^{3}$. The endurance and efficiency of these motors need to be improved.

Physical micromotors are propelled by varying fields. For instance, a helix of magnetic material spins around its axis under a rotating magnetic field. These devices are easier to control: changing the field's orientation and frequency alters the direction and speed of the motor. Such

'magnetic swimmers' mimic flagella, the tails that propel some microorganisms ${ }^{4}$. Ultrasound, too, can be used for propulsion and guidance ${ }^{5}$.

These micromotors have less thrust than the chemical motors and need complicated actuation systems. They hold promise for carrying cargo (sensors, drugs and genetic therapies), for capturing and transporting cells and for performing microsurgery and biopsies $^{1}$.

Biohybrid micromotors combine a biological agent such as a bacterium, muscle or sperm cell with a synthetic part. They can be directed by external fields or by the cells and microorganisms themselves, as they move, sense and respond to biochemicals, acidity or magnetic fields. For example, bacteria that perceive

\section{"Combinations of materials, microorganisms, microstructures and functions all need to be tested. together in vivo."}

Earth's magnet-

ism have been explored as potential drug carriers in blood vessels ${ }^{6}$. Biohybrid swimmers may travel naturally through the body. They can pass through tissues to deliver drugs deeply and can stimulate reactions such as those involved in fertilization.

For example, we have demonstrated how a motile sperm cell, loaded with a drug, could be coupled to a magnetic microstructure that guides and then releases the sperm-drug complex to potentially treat cancers in the reproductive tract ${ }^{7}$. And we have used rotating magnets to drive a helixshaped physical micromotor to deliver a live but immotile bovine sperm cell to an oocyte (egg). Such 'spermbots' could lead to new assisted-reproduction techniques ${ }^{8,9}$. Low sperm count and motility are the two main causes of male infertility, accounting for $40 \%$ of all cases. If spermbots can capture and guide sperm to an oocyte to fertilize it in vivo, this should result in higher fertilization rates, procedures that are less invasive, and more-natural conditions for the developing embryo.

\section{PRACTICAL CHALLENGES}

All three micromotor types share challenges. The materials they are made from must be proved to be biocompatible (such as polymers; metals including gold and zinc; proteins and DNA) or biodegradable (alginate, gelatin, calcium carbonate). They need to be able to perform a wide range of tasks: from sensing and responding to their environment to storing and delivering molecules or cells when stimulated by physical cues or by certain molecules, disease biomarkers, temperatures or levels of acidity. They need to be more manoeuvrable in three dimensions, in viscous and elastic body fluids and in phantom organs. And their targeting must be accurate.

Before any of these tiny vehicles can be used in vivo, we need to plan how to remove or stop them. They might be driven back to the starting point (mouth, eyes, ear, vagina, urethra), but this could be tedious, especially when many have been introduced. They could degrade, with the products absorbed or expelled naturally, as with tissue-engineering scaffolds, for instance. Biodegradable materials such as chitosan,

\section{THREE MICROMOTOR PROTOTYPES}

Tiny machines and motors micrometres in size and driven by chemical reactions, physical

fields or cells could deliver drugs, guide reactions or perform surgery in the body.

CHEMICAL

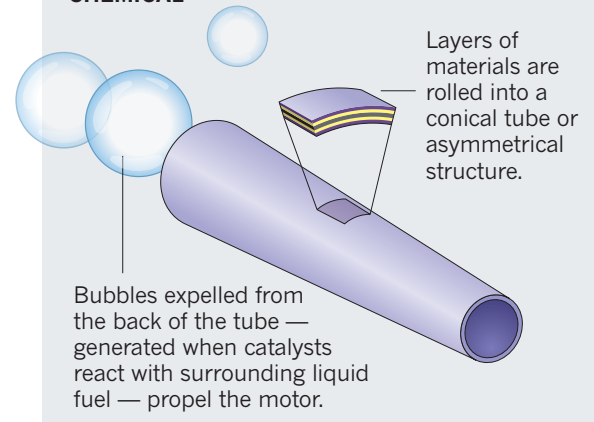

PHYSICAL

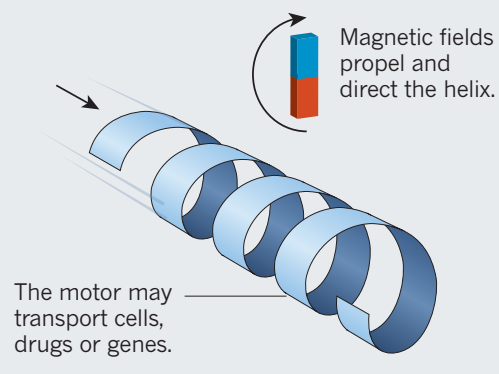

BIOHYBRID

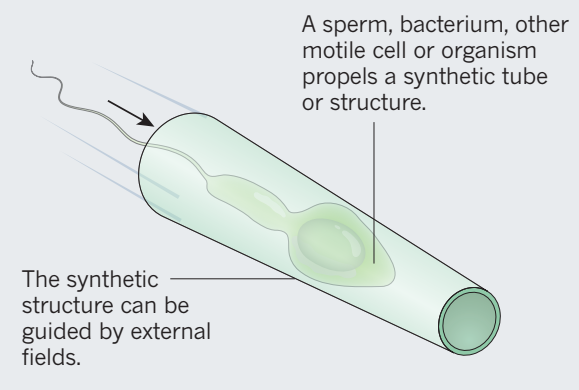

POTENTIAL MEDICAL USES

Drug delivery; sensing; cell transport; microsurgery and biopsies; assisted fertilization;

gene transfections; cancer treatment; lab-on-chip applications 
polylactic acid or polyacrolactone dissolve at a certain $\mathrm{pH}$, temperature or time. But small amounts of magnetic substances, metals or oxides will also be present, and their degradation and toxicity need to be studied. Stable biorobots could remain in the body as implants, monitoring the function of an organ, say.

Regulation lags behind research. Whereas active micromotors are far from being applied in clinics, some passive micro and nanoscale therapeutics have been approved. For example, silver nanoparticles are used as antibacterial wound dressings. Therapeutics that encapsulate drugs within cells or use cellular processes to modify genes or deliver drugs could be made more targeted and personalized, if more were known about their side effects.

In the United States, live biotherapeutic products, including some vaccines, are regulated by the US Food and Drug Administration and must pass a barrage of tests in animals and humans. Blends of live and synthetic components will be harder to assess. Combinations of materials, microorganisms, microstructures and functions all need to be tested together in vivo.

\section{SEEING FURTHER}

Tracking the devices in vivo is crucial. Current imaging techniques, such as radiology, ultrasound, infrared and magnetic resonance imaging (MRI) are too coarse, insensitive and slow to find, let alone follow, micromotors operating deep within the body. The radioactive isotopes used in radiology and nuclear medicine are hazardous in high concentrations and when used for a prolonged time. Normal clinical MRI (with magnetic field strengths of up to 3 tesla) can resolve structures that are around 300 micrometres across - good enough to image blood vessels. Higher magnetic fields (10-12 tesla) can resolve 100 micrometres, but require expensive infrastructure. MRI scans take seconds to acquire and their resolution worsens when sequences are sped up.

A new method is called for. Ideally, it should be capable of imaging, in $3 \mathrm{D}$, micromotors that are about 10 centimetres below the skin. It must resolve devices 1-50 micrometres across. And it must track them moving at minimum speeds of tens of micrometres per second - typical of bacteria or sperm - and ideally more to an accuracy of milliseconds for hours.

There are promising developments. Bioimaging researchers are manipulating light, sound and electromagnetic waves to minimize the two main effects that blur images: diffraction and scattering. Sensitivity and exposure times depend mainly on contrast. This can be enhanced by applying to the target cells or devices chemical agents

that darken or fluoresce when stimulated (such as quantum dots). Ultrasound signals might be boosted through the use of small reflectors.

Combinations of these techniques look most encouraging, in our view. For example, Christian Wiest and his colleagues at iThera Medical in Munich, Germany, are developing multispectral optoacoustic tomography, which exploits the best attributes of infrared and ultrasound imaging. When laser pulses are fired at tissues, they expand and contract, giving off ultrasonic pressure waves that can be turned into a $3 \mathrm{D}$ image. These images have high contrast (governed by the absorption of light) and high spatial resolution (ultrasound scatters very little). Frequencies of light or ultrasound can be chosen to make certain molecules glow or darken. Such approaches can now reach resolutions of about 150 micrometres at depths of about $2-3$ centimetres ${ }^{10}$. With focused research, they could become good enough to track microbots within a few years.

Cutting-edge ultrasound methods are also improving rapidly. Holography - encoding a light field as an interference pattern in a photograph - is a promising concept

"With a coordinated push, microbots could usher in an era of non-invasive therapies within adecade." for both imaging and control of microobjects ${ }^{11}$. And our research group is exploring whether the direction and velocity of microbots can be tracked by measuring the reflec-

tion, transmission or emission of certain frequencies of infrared light as a function of wavelength and time. Ultimately, several approaches may be needed.

\section{MOTORING FORWARD}

Over the next two years, the field needs to prepare for when the visualization systems become good enough to start testing and tracking active therapies in live animals.

Microbot researchers need to establish mechanisms for operating microbots, possibly even in swarms, inside the body. For example, ultrasound and magnetic fields could direct them broadly to the right region, from where finer, biochemical sensing would take over. The goal is a microbot that can sense, diagnose and act autonomously, while people monitor it and retain control in case of malfunction.

Research funders and universities need to support such cross-disciplinary work. Most of our activities are carried out within a nationwide priority programme called 'Microswimmers' that is funded by the DFG, one of Germany's main research-funding agencies.

Regulators and ethics panels should establish requirements for micromotor and biohybrid therapies. The longterm toxicity and immunoreactions of biodegradable materials and their functional coatings (such as metals, oxides and polymers) require exhaustive testing.

Clinicians should ask how these new materials and technologies should be harnessed to understand processes in the body and to design treatments. Which applications are most amenable to microbot therapies? How might microscopic tissue interventions actually be performed?

Regulatory restrictions mean that biohybrids will first be explored in lab-onchip systems for biochemical sensing and immunoassay performance. But we have asked some clinicians how they see spermbots being used in their practices. Dunja Baston-Büst at Germany's University Hospital Düsseldorf, for instance, agrees that spermbots might be useful for delivering drugs or genes into the female reproductive tract to treat cancers or diseases of the oocyte. And Elkin Lucena from the Colombian Center of Fertility and Sterility (CECOLFES) in Bogotá thinks that if all the challenges can be overcome, microbot fertilization could eventually become an alternative to in vitro techniques such as injecting a sperm into an egg.

With a coordinated push, microbots could usher in an era of non-invasive therapies within a decade.

Mariana Medina-Sánchez is group leader of the Micro and Nano-biomedical Engineering Group and Oliver G. Schmidt is director of the Institute for Integrative Nanosciences at the Leibniz IFW in Dresden, Germany. Oliver G. Schmidt is also Professor of Material Systems for Nanoelectronics at Technische Universität Chemnitz, Germany.

e-mails:m.medina.sanchez@ifw-dresden. de;o.schmidt@ifw-dresden.de

1. Guix, M., Mayorga-Martinez, C. C. \& Merkoçi, A. Chem. Rev. 114, 6285-6322 (2014)

2. Ma, X., Hortelão, A. C., Patiño, T. \& Sánchez, S. ACS Nano 10, 9111-9122 (2016).

3. Gao, W. et al. ACS Nano 9, 117-123 (2015).

4. Qiu, F. \& Nelson, B. J. Engineering 1, 021-026 (2015).

5. Rao, K. J. et al. Small 11, 2836-2846 (2015).

6. Martel, S., Mohammadi, M., Felfoul, O., Lu, Z. \& Pouponneau, P. Int. J. Rob. Res. 28, 571-582 (2009).

7. Xu, H. et al. Preprint at https://arxiv.org/ abs/1703.08510 (2017).

8. Medina-Sánchez, M., Schwarz, L., Meyer, A. K., Hebenstreit, F. \& Schmidt, O. G. Nano Lett. 16, 555-561 (2016)

9. Magdanz, V., Sanchez, S. \& Schmidt, O. G. Adv. Mater. 25, 6581-6588 (2013).

10.Deán-Ben, X. L., Gottschalk, S., Mc Larney, B., Shoham, S. \& Razansky, D. Chem. Soc. Rev. 46, 2158-2198 (2017).

11.Melde, K., Mark, A. G., Qiu, T. \& Fischer, P. Nature 537, 518-522 (2016). 